\title{
Usefulness of three-dimensional echocardiography for the assessment of ventricular function in children: Comparison with cardiac magnetic resonance, with a focus on patients with arrhythmia
}

\author{
Halszka Kamińska ${ }^{1}$, Łukasz A. Małek ${ }^{2}$, Marzena Barczuk-Falęcka ${ }^{3}$, Bożena Werner ${ }^{1}$
}

${ }^{1}$ Department of Pediatric Cardiology and General Pediatrics, Medical University of Warsaw, Poland

${ }^{2}$ Faculty of Rehabilitation, University of Physical Education, Warsaw, Poland

${ }^{3}$ Department of Pediatric Radiology, Medical University of Warsaw, Poland

\begin{abstract}
Background: Focusing on patients with arrhythmia, the aims of this study was to assess ventricular function in children using three-dimensional echocardiography (3D-ECHO) and to compare the results to those obtained with cardiac magnetic resonance (CMR).

Methods: The study group consisted of 43 children in whom 3D-ECHO and CMR were performed. Twenty-five patients had a ventricular arrhythmia, 7 left ventricular cardiomyopathies, 9 proved to be healthy. In all children, $3 D$-ECHO (offline analysis) was used to assess ventricular ejection fraction $(E F)$. The results were compared to CMR using the Bland-Altman analysis and linear regression. The Student paired T-test was used to compare of means between both modalities.

Results: The relation between the results derived from both methods is linear (for left ventricle: estimated slope $=1.031, p<0.0001, R$-squared $=0.998$; for right ventricle: estimated slope $=0.993$, $p<0.0001, R$-squared $=0.998)$. In spite of minimal mean differences between results for both ventricles and narrow $95 \%$ confidence intervals, the paired $t$-test proved those differences not to be significant $(p>0.05)$ for the right ventricle but statistically significant $(p<0.05)$ for the left ventricle, for which the left ventricular EF calculated in $3 D-E C H O$ was systematically underestimated with a mean difference of $-1.8 \% \pm 2.6 \%(p<0.0001)$.

Conclusions: Three-dimensional echocardiography assessment of both left and right ventricular EF in children showed high significant correlation and agreement with CMR. 3D-ECHO could be a valuable tool in follow-up of children with arrhythmic disorders requiring regular assessment of ventricular function. (Cardiol J 2021; 28, 4: 549-557)

Key words: three-dimensional echocardiography, cardiac magnetic resonance, ventricular ejection fraction, children, arrhythmia
\end{abstract}

\section{Introduction}

Arrhythmias are considered one of the most significant problems of modern pediatric cardiology. As in the majority of children, no connection to specific structural or functional cardiac abnormality can be found, those cases often are defined as idiopathic. The use of three-dimensional echocardiography (3D-ECHO) for non-invasive assessment of ventricular function is at present widespread and

Address for correspondence: Prof. Bożena Werner, MD, PhD, Head of Department of Pediatric Cardiology and General Pediatrics, Medical University of Warsaw, ul. Żwirki i Wigury 63A, 02-091 Warszawa, Poland, tel: +48 223179588 , e-mail: bozena.werner@wum.edu.pl

Received: 18.01.2019 Accepted: 14.02.2019

This article is available in open access under Creative Common Attribution-Non-Commercial-No Derivatives 4.0 International (CC BY-NC-ND 4.0) license, allowing to download articles and share them with others as long as they credit the authors and the publisher, but without permission to change them in any way or use them commercially. 
universal, especially in adult patients. In pediatric cardiology the method was embraced much later and its exploit is still noncompliant with potential benefits. Being close in accuracy although more feasible and less expensive than cardiac magnetic resonance (CMR) 3D-ECHO may be valuable diagnostic tool especially in groups of children demanding regular assessment of ventricular function.

The aim of the study was to assess ventricular function in children using 3D-ECHO and compare the results to those obtained with CMR, focusing especially on patients with arrhythmia.

\section{Methods}

The prospective study included 43 consecutive children hospitalised in the Pediatric Cardiology Department, aged 4 months to 17 years, average $13.7 \pm 3.8$ years, in whom both $3 \mathrm{D}-\mathrm{ECHO}$ and CMR were performed due to clinical indications. In all the children electrocardiography (ECG) and 24-hours ECG Holter monitoring were used in diagnostics. In this group, 27 patients suffered from arrhythmia: two supraventricular (one with single extrasystolic beats, one with nodal rhythm), 25 ventricular arrhythmias (in all cases at least one of extrasystolic morphologies was left bundle branch block) classified as mild in 9 patients (single monomorphic beats, less than $20 \%$ of arrhythmia in 24 -h Holter-ECG monitoring) and severe in 16 children (defined as complex with ventricular tachycardia or over $20 \%$ single beats during $24 \mathrm{~h}$ ). Out of 25 children, in 20 ventricular arrhythmias were classified as idiopathic (no evident source in cardiac morphology or function was detected), three patients fulfilled the criteria of arrhythmogenic right ventricular cardiomyopathy (ARVC), one was diagnosed with Andersen-Tawill syndrome, and in one arrhythmia occurred after surgical repair of ventricular septal defect. Seven patients of the studied cohort were diagnosed with left ventricular cardiomyopathies, 9 patients proved to be healthy either in the course of diagnostics for suspected myocarditis (based on elevated plasma troponin level and/or abnormalities in ECG) or after completed healing process. The characteristics of the studied group are presented in Table 1 .

In all patients $3 \mathrm{D}-\mathrm{ECHO}$ and $\mathrm{CMR}$ were performed. In one child ejection fraction (EF) of both ventricles and in one - the right ventricle could not be calculated in CMR due to artefacts connected with excessive arrhythmia. Those patients were not included in the statistical analysis, leaving 42 children for the analysis of left and 41 - right
Table 1. Characteristics of the studied group.

\begin{tabular}{|c|c|}
\hline Patients' characteristics & Details \\
\hline Age [years] & $13.7 \pm 3.8$ \\
\hline \multicolumn{2}{|l|}{ Sex: } \\
\hline Boys & 26 \\
\hline Girls & 17 \\
\hline $\mathrm{BSA}\left[\mathrm{m}^{2}\right]$ & $\begin{array}{c}0.56-2.04 ; \\
\text { mean } 1.61 \pm 0.34\end{array}$ \\
\hline \multicolumn{2}{|l|}{ Diagnosis: } \\
\hline Arrhythmia & 2 \\
\hline Supraventricular & 25 \\
\hline \multicolumn{2}{|l|}{ Ventricular } \\
\hline \multicolumn{2}{|l|}{ Severity } \\
\hline Mild $(<20 \% / 24 \mathrm{~h}+$ no VT) & 9 \\
\hline $\begin{array}{l}\text { Severe (> } 20 \% / 24 \mathrm{~h} \\
\text { and/ or VT) }\end{array}$ & 16 \\
\hline \multicolumn{2}{|l|}{ Cause } \\
\hline Idiopathic & 20 \\
\hline ARVC & 3 \\
\hline VSD (after operation) & 1 \\
\hline ATS & 1 \\
\hline LV cardiomyopathy: & 7 \\
\hline DCM & 3 \\
\hline $\mathrm{HCM}$ & 2 \\
\hline $\mathrm{RCM}$ & 1 \\
\hline NCLV & 1 \\
\hline Healthy subjects* & 9 \\
\hline
\end{tabular}

*Initially suspected or successfully treated myocarditis; ARVC - arrhythmogenic right ventricular cardiomyopathy; ATS - Andersen-

-Tawill syndrome; BSA — body surface area; DCM — dilated cardiomyopathy; HCM — hypertrophied cardiomyopathy; NCLV noncompacted left ventricle; RCM - restrictive cardiomyopathy; VSD - ventricular septal defect

ventricular function. The postprocessing (offline analysis) of echocardiography data was obtained without knowing the results of CMR.

\section{D-ECHO: Image acquisition}

All patients underwent standard echocardiography examination (Philips EPIQ system, Netherlands) during which the ECG-gated 3D full-volume data sets were recorded using a matrix X5-1/X7-2 transducer from an apical window in the patients' left lateral decubitus position, possibly while withholding breath. Four consecutive cardiac cycles were registered to obtain optimal resolution. In 2 patients with excessive arrhythmia we recorded only two cardiac cycles and the data proved adequate for further analysis. The left and right ventricles were addressed separately to assure that the data set would contain the whole chamber 
with its apical portion and the widest diameter of concordant atrioventricular valve. For the left ventricle standard 4-chamber view was used; for the right ventricle the probe was moved slightly to the left side with its tail tilted posteriorly and counter clockwise to open the apical part and include the full capacity of outflow track in the pyramidal data set. For both ventricles the probe's position and its spatial orientation was controlled by a simultaneous 2-dimensional view of coronal and sagittal planes. At least three full-volume acquisitions for each ventricle were recorded in each patient; the one with the highest quality was assigned for later post processing. In patients with sinus rhythm the recording time approached $3 \mathrm{~min}$, although in patients with arrhythmia it was considerably longer (mean $5 \mathrm{~min}$ ) because recording of four consecutive sinus beats with no extrasystoly in real time was more challenging.

\section{Postprocessing}

Full-volume 3D digital data sets for both left and right ventricle were exported to an external server for offline analysis using dedicated software (TomTec Imaging Systems GMBH, Germany; Image Arena 4.6). For the left ventricle 4D LVAnalysis software was used and for the right ventricle $-4 \mathrm{D}$ RV-Function module. For each ventricle analysis, the user identified specific landmarks in end-diastolic and end-systolic views (apex, mitral, and aortic annulus for left ventricle, and additionally ventricular diameter in short axis view along with interventricular septum perimeters for the right ventricle). Based on the landmarks, semiautomatic tracing of endocardium was performed and corrected manually by the software operator. The analysis was performed using high-contrast monitor settings which, from experience, helps to define clearer line to track while manual correction of contour is made. Trabeculae and papillary muscles into ventricular cavity was included. For optimal quality of full-volume data sets the analysis of both ventricles was completed in a mean time of $4 \mathrm{~min}$, and up to $9 \mathrm{~min}$ for images of poorer quality requiring more manual tracing. The results of the analysis being systolic function of both ventricles illustrated by EF were automatically calculated from end-diastolic volume (EDV), and end-systolic volume (ESV). A graphic presentation of the results is featured in Figure 1A, B.

\section{Cardiac magnetic resonance}

All CMR studies were acquired with a Siemens Magnetom Skyra 3 Tesla scanner (Siemens, Erlan- gen, Germany). Five patients who were unable to cooperate during the procedure (the youngest or hyperactive children) required general anaesthesia. A routinely used CMR protocol to assess left and right ventricular size and function included initial scout images followed by cine steady-state free precession (SSFP) breath hold sequences in 2-, 3 -, and 4-chamber views to set up final imaging planes and a stack of short-axis images from the atrioventricular annulus to the apex. Imaging parameters were as follows: field of view $340 \mathrm{~mm}$, matrix 208, repetition time approximately $39.24 \mathrm{~ms}$, echo time $1.43 \mathrm{~ms}$, flip angle 39 degrees, slice thickness $6-8 \mathrm{~mm}$ (depending on the child age), gap $2 \mathrm{~mm}$, in-plane image resolution $1.6 \times 1.6 \times$ $\times 6-8 \mathrm{~mm}$, and temporal resolution 25 phases per cardiac cycle $[1,2]$. Images were analysed with the use of a dedicated software. Initially, short-axis SSFP cine images were previewed from the base to the apex in a cinematic mode, then endocardial contours for end-diastole and end-systole of both ventricles were manually traced. Trabeculae and papillary muscles were considered as ventricle cavities. Delineated contours were used for the quantification of ventricular ejection fractions (left ventricular EF [LVEF], and right ventricular $\mathrm{EF}$ [RVEF]). The mean time of image acquisition was $40 \mathrm{~min}$ (longer in children requiring general anaesthesia - up to $1.5 \mathrm{~h}$ ). The mean time of image analysis was $20 \mathrm{~min}$.

\section{Statistical analysis}

Bland-Altman analysis and linear regression were used to compare results of LVEF and RVEF obtained with 3D-ECHO against CMR acknowledged as the method of reference. A p-value of less than 0.05 was considered statistically significant [3]. For the whole cohort and selected subgroups of patients with arrhythmia the mean differences between $3 \mathrm{D}-\mathrm{ECHO}$ and CMR results were calculated and the $95 \%$ confidence intervals (CIs) determined. Because data were normally distributed (verified by the Shapiro-Wilk test), a paired Student t-test was used to compare means of LVEF and RVEF results obtained from 3D-ECHO and CMR, again using CMR as the method of reference.

All calculations and graphs were made in the $\mathrm{R}$ software version 3.3.1. (distributed under the terms of the GNU General Public License).

The study was approved by the Medical University's Ethics Committee. In all patients, written informed consent was obtained from parents and for children older than 16 years of age. 


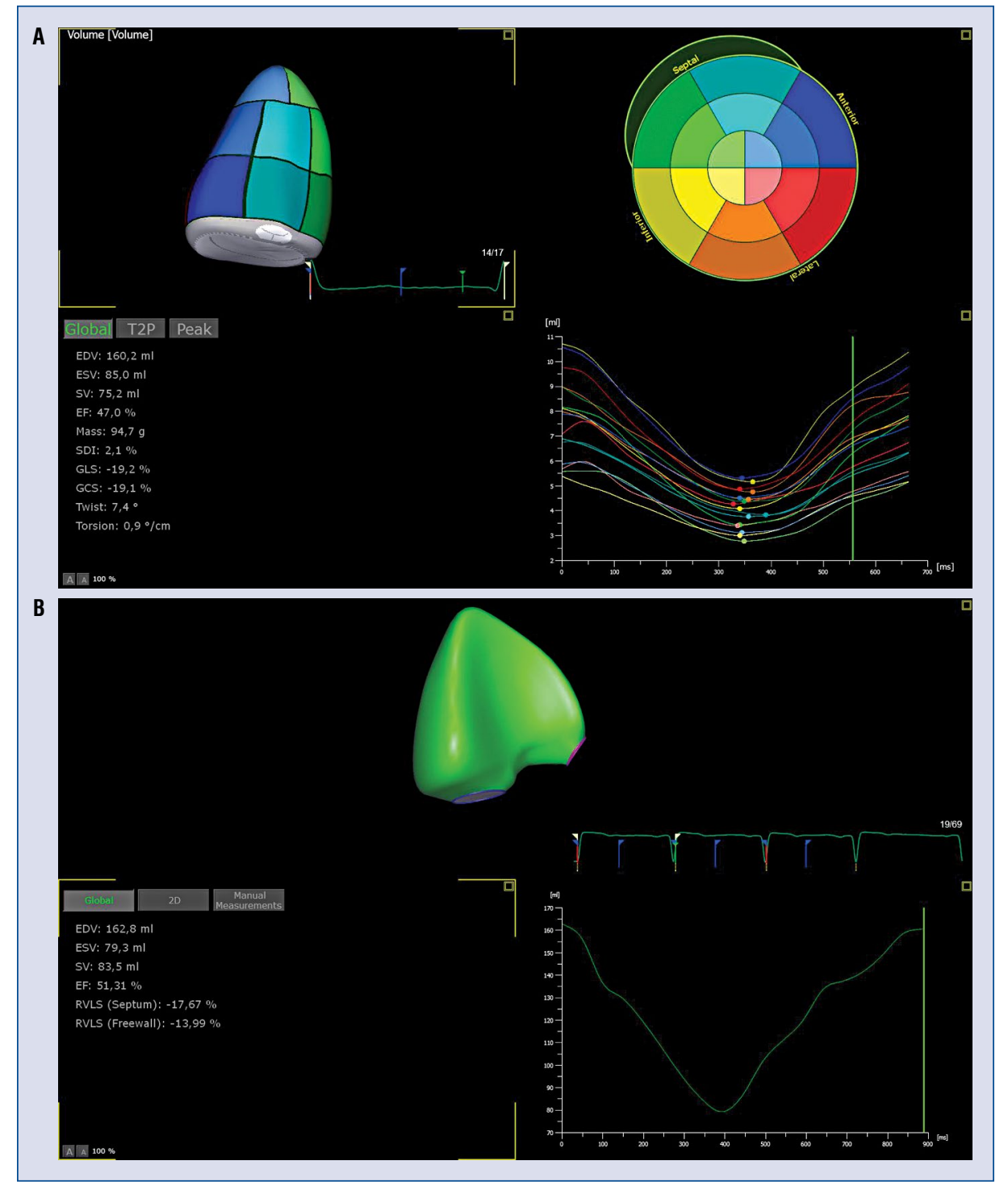

Figure 1. Three-dimensional echocardiography offline analysis; A. Left ventricular model with calculated ejection fraction; B. Right ventricular analysis results.

\section{Results}

Bland-Altman plot proved minor mean differences with narrow limits of agreement between the results obtained with $3 \mathrm{D}-\mathrm{ECHO}$ and $\mathrm{CMR}$ for both LVEF and RVEF, marking very high significant correlation (for LVEF: $r=0.903, p<0.00001$; for RVEF: $\mathrm{r}=0.966, \mathrm{p}<0.00001)$ and agreement (Fig. 2A, B). The relation between the results derived from both methods is linear, and it can be approximated by the identity function (Fig. 3A, B).

For LVEF, the estimated slope was 1.031, standard error $=0.007(\mathrm{p}<0.00001)$, R-squared $=$ $=0.998$; the average width of $95 \%$ prediction interval was $10.6 \% \pm 5.3 \%$. Comparing LVEF calculated with 3D-ECHO to reference CMR values - in 34\% of patients the difference was lower than $1 \%$, in $59 \%$ under $2 \%$, in $68 \%$ under $3 \%$, and in $78 \%$ below $4 \%$. However, the paired Student t-test showed a statistically significant difference between means of LVEF calculated in 3D-ECHO and CMR - both for the whole cohort and in subgroups with arrhythmia ( $\mathrm{p}<0.005)$. 3D-ECHO results proved to be minimally underestimated comparing to CMR results with mean difference of $-1.8 \pm 2.6 \%$ $(\mathrm{p}<0.0001)$ for the whole population, in the subgroup with arrhythmia $-2.3 \pm 2.5 \%(\mathrm{p}<0.0001)$, in children with severe ventricular arrhythmia $-3.1 \pm 2.7 \%(\mathrm{p}=0.0006)$, and in patients with severe idiopathic ventricular arrhythmia $-2.6 \pm$ 

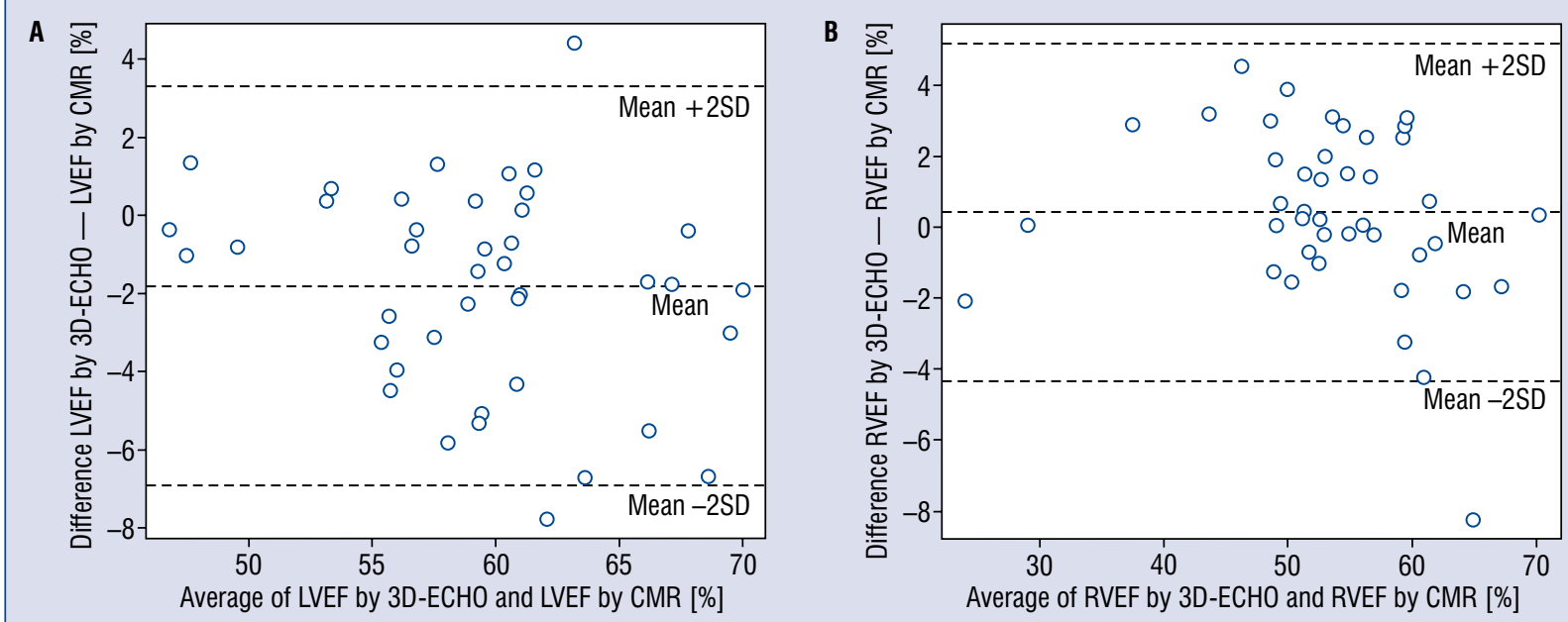

Figure 2. Bland-Altman analysis. Left $(\mathbf{A})$ and right $(B)$ ventricular ejection fraction (LVEF, RVEF, respectively): mean differences between results of three-dimensional echocardiography (3D-ECHO) and cardiac magnetic resonance (CMR) with limits of agreement.
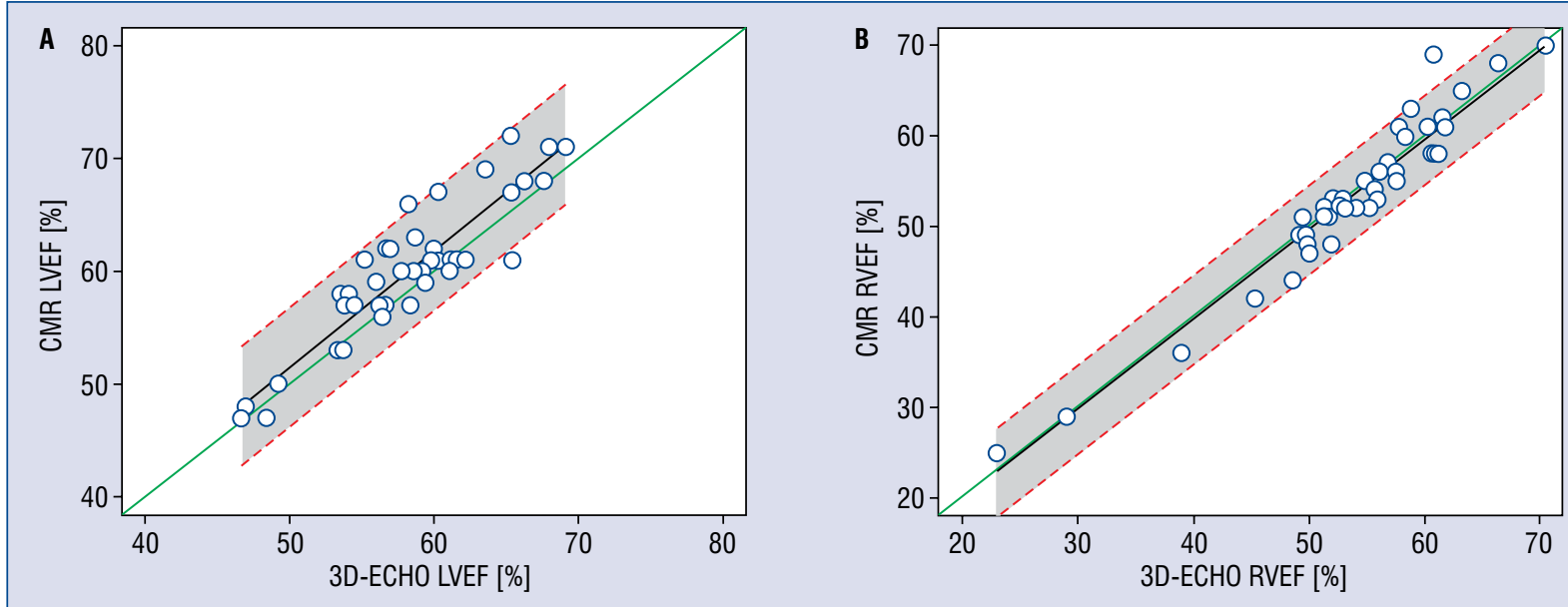

Figure 3. Identity function comparing results of left $(\mathbf{A})$ and right $(\mathbf{B})$ ventricular ejection fraction (LVEF, RVEF, respectively) between three-dimensional echocardiography (3D-ECHO) and cardiac magnetic resonance (CMR). Regression of $\mathrm{Y}(\mathrm{CMR})$ on $\mathrm{X}(3 \mathrm{D}-\mathrm{ECHO})$, with prediction limits. A. The average width of prediction interval $=10.6 \pm 5.3 \%$; $\mathrm{B}$. The average width of prediction interval $=10.0 \pm 5.0 \%$.

$\pm 2.8 \%(\mathrm{p}=0.0088)$. At the same time the $95 \%$ CIs for the whole group studied and arrhythmic subgroups proved to be narrow, marking good consistency of both methods. Also, those underestimations are considered minimal and irrelevant for single patients in clinical practice. The results are presented in Table 2 .

For RVEF the estimated slope was 0.993, standard error $=0.007(\mathrm{p}<0.00001), \mathrm{R}$-squared $=0.998$; average width of $95 \%$ prediction interval was $10.0 \pm 5.0 \%$. Comparison of RVEF from
3D-ECHO to CMR values in $39 \%$ of patients showed the difference below $1 \%$, in $63 \%$ under $2 \%$, in $78 \%$ under $3 \%$, and in as much as $93 \%$ below $4 \%$. Almost perfect agreement was obtained between results of 3D-ECHO and CMR with the paired Student t-test proving the lack of a statistically significant difference between mean values of RVEF calculated in 3D-ECHO and CMR marked by $\mathrm{p}>0.05$ for the whole cohort and in arrhythmic subgroups. The mean difference between $3 \mathrm{D}-\mathrm{ECHO}$ and CMR results was $0.4 \pm 2.4 \%$ for the whole cohort $(\mathrm{p}=0.29)$, 
Table 2. Results of left ventricular ejection fraction (LVEF) measurements in three-dimensional echocardiography (3D-ECHO) and cardiac magnetic resonance (CMR) in subgroups of patients: ranges and mean values with standard deviations.

\begin{tabular}{|c|c|c|c|c|}
\hline LVEF [\%] & 3D-ECHO (n = 42) & CMR (n = 42) & $95 \% \mathrm{Cl}^{*}$ & $\mathbf{P}$ \\
\hline \multirow[t]{2}{*}{ Study group } & Range: 46.6-69.1\% & Range: $47.0-72.0 \%$ & \multirow{2}{*}[-2.6,-1.0]{} & \multirow{2}{*}{$<0.0001$} \\
\hline & Mean: $58.5 \pm 5.4 \%$ & Mean: $60.3 \pm 6.0 \%$ & & \\
\hline \multirow[t]{2}{*}{ Arrhythmia (26) } & Range: 49.2-69.1\% & Range: $50.0-71.0 \%$ & \multirow{2}{*}[-3.3,-1.3]{} & \multirow{2}{*}{$<0.0001$} \\
\hline & Mean: $59.0 \pm 4.7 \%$ & Mean: $61.2 \pm 5.0 \%$ & & \\
\hline \multirow[t]{2}{*}{ Severe ventricular arrhythmia (15) } & Range: $49.2-68.0 \%$ & Range: $50.0-71.0 \%$ & \multirow{2}{*}[-4.6,-1.6]{} & \multirow{2}{*}{0.0006} \\
\hline & Mean: $57.9 \pm 4.5 \%$ & Mean: $60.7 \pm 5.2 \%$ & & \\
\hline \multirow{2}{*}{$\begin{array}{l}\text { Severe idiopathic ventricular } \\
\text { arrhythmia (12) }\end{array}$} & Range: $49.2-68.0 \%$ & Range: $50.0-71.0 \%$ & \multirow{2}{*}[-4.4,-0.8]{} & \multirow{2}{*}{0.0088} \\
\hline & Mean: $58.4 \pm 4.8 \%$ & Mean: $60.7 \pm 5.8 \%$ & & \\
\hline ARVC (3) & Range: $53.5-58.7 \%$ & Range: $58.0-63.0 \%$ & \multicolumn{2}{|c|}{$\begin{array}{l}\text { Too small sample for } \\
\text { statistical testing }\end{array}$} \\
\hline
\end{tabular}

*Difference = ejection fraction [\%] measured by 3D-ECHO - ejection fraction [\%] measured by CMR; ARVC - arrhythmogenic right ventricular cardiomyopathy; $\mathrm{Cl}$ - confidence interval

Table 3. Results of right ventricular ejection fraction (RVEF) measurements in three-dimensional echocardiography (3D-ECHO) and cardiac magnetic resonance (CMR) in subgroups of patients: ranges and mean values with standard deviations.

\begin{tabular}{lcccc}
\hline RVEF [\%] & 3D-ECHO $(\mathbf{n}=41)$ & CMR $(\mathbf{n}=41)$ & 95\% Cl* & P \\
\hline Study group & Range: $22.9-70.3 \%$ & Range: $25.0-70.0 \%$ & {$[-0.3,+1.2]$} & 0.29 \\
& Mean: $54.5 \pm 8.8 \%$ & Mean: $53.6 \pm 9.4 \%$ & & 0.43 \\
Arrhythmia (25) & Range: $22.9-70.3 \%$ & Range: $25.0-70.0 \%$ & {$[-0.5,+1.2]$} & \\
& Mean: $52.8 \pm 9.8 \%$ & Mean: $52.0 \pm 10.1 \%$ & & 0.46 \\
Severe ventricular arrhythmia (14) & Range: $22.9-70.3 \%$ & Range: $25.0-70.0 \%$ & {$[-0.8,+1.7]$} \\
& Mean: $50.5 \pm 11.9 \%$ & Mean: $49.1 \pm 11.9 \%$ & & 0.48 \\
Severe idiopathic ventricular & Range: $48.5-70.3 \%$ & Range: $44.0-70.0 \%$ & {$[-1.0,+2.0]$} \\
arrhythmia (12) & Mean: $55.2 \pm 6.4 \%$ & Mean: $53.9 \pm 7.1 \%$ & Too small sample for \\
ARVC (3) & Range: $22.9-39.0 \%$ & Range: $25.0-36.0 \%$ & statistical testing \\
\hline
\end{tabular}

*Difference = ejection fraction [\%] measured by 3D-ECHO - ejection fraction [\%] measured by CMR; ARVC — arrhythmogenic right ventricular cardiomyopathy; $\mathrm{Cl}$ — confidence interval

$0.3 \pm 2.2 \%$ in the subgroup with arrhythmia $(\mathrm{p}=$ $=0.43), 0.5 \pm 2.3 \%$ in the subgroup with severe ventricular arrhythmia $(\mathrm{p}=0.46)$ and $0.5 \pm 2.4 \%$ for patients with severe ventricular arrhythmia classified as idiopathic $(\mathrm{p}=0.48)$. The $95 \% \mathrm{CI}$ for the whole group studied and arrhythmic subgroups proved to be narrow, again marking close agreement with CMR results. The data are presented in Table 3.

\section{Discussion}

Arrhythmias are considered one of the most prominent problems in pediatric cardiology [4].
Contrary to the adult population, most cases of arrhythmia in children are classified as idiopathic because no link to cardiac morphology or evident haemodynamic dysfunction can be found [5-8]. In most cases the risk is low and general prognosis is good, patients do not usually require pharmacotherapy, even if extrasystole is common. However, for some arrhythmias the risk of sudden cardiac death is much higher. In diagnostics of ARVC enlarged chamber and deteriorated function of the right ventricle are among the most prominent major criteria, providing in fact half of the definitive diagnosis $[9,10]$. The progression of the disease, including deterioration of ventricular function, 
may be gradual and diffused in time [11-13]. For that reason, the accurate and regular assessment of ventricular function is crucial in patients with ventricular arrhythmia.

Unfortunately, widely accessible and inexpensive two-dimensional echocardiography (2D-ECHO) offers very biased data because a singleplane view cannot illustrate the complex morphology of the right ventricle [14-17]. CMR is much more accurate in this assessment and is considered the gold standard in calculating ventricular volume and function. Additionally, it offers information about potential myocardial fibrosis or fatty infiltration (helpful in establishing the diagnosis of ARVC, although not included in diagnostic criteria). Unfortunately, its low accessibility and high cost prevent it from being a truly universal tool, especially in patients with severe arrhythmia in whom initially normal right ventricular parameters might yet evolve into cardiomyopathy with time and need to be measured regularly [14]. Furthermore, the use of CMR has significant limitations in a group of infants and small children as well as patients with hyperactivity or anxiety disorders. The procedure is lengthy (at least $40 \mathrm{~min}$ ) and during that time the patient has to lie still and hold breath on demand; that alone creates a cooperative problem in the pediatric population. For this reason, many patients ( 5 in the present study cohort) required general anesthesia with all its potential risks. Another group of patients in whom assessment of ventricular volume and function might be challenging is the population with excessive arrhythmia (especially with numerous extrasystolic beats), because the method is ECG-gated and requires 10 consecutive regular (sinus) beats to obtain optimal image resolution. Among the present study group, 2 children with multiple extrasystole, acquisition of optimal images was impossible.

Three-dimensional echocardiography, with its rapid evolution during the last three decades, seems to be an accessible and inexpensive tool for the assessment of cardiac function in clinical practice because it combines the accuracy of magnetic resonance imaging with the already high and constantly expanding accessibility and cost effectiveness of two-dimensional ultrasound systems [18-22]. The image acquisition is faster than CMR and requires fewer consecutive regular heart beats to produce data of adequate quality.

In the current study, focus was concentrated on evaluating the accuracy of $3 \mathrm{D}-\mathrm{ECHO}$ in accessing ventricular function in children in comparison to CMR as the modality of reference.
In the group of children studied, results of both LVEF and RVEF calculated in 3D-ECHO proved to have a very high correlation and agreement with the data obtained in CMR. This consistency was proven both for the whole cohort and within extracted subgroups - patients with arrhythmic disorders in general, severe ventricular arrhythmias, and among cases of arrhythmia classified as idiopathic.

The mean differences between values of ventricular systolic function (LVEF and RVEF) calculated in 3D-ECHO and CMR were minimal with narrow (<4\%) 95\% CI. However, while for the right ventricle the consistency of results between both methods was proven to be almost perfect in the paired Student $t$-test $(\mathrm{p}>0.05$ interpreted in this case as a lack of significant difference), LVEF proved to be minimally underestimated by $3 \mathrm{D}$ -ECHO in the whole population and arrhythmic subgroups with the highest mean difference of $-3.1 \pm$ $\pm 2.7 \%$ in the group of patients with severe ventricular arrhythmia. That difference was proven to be statistically significant $(\mathrm{p}<0.05)$ by the paired Student t-test although irrelevant in clinical practice. The small 95\% CI for mean difference pointed to close agreement with CMR results. This tendency is coherent with data published so far [23].

Published studies have shown good to excellent correlation between LVEF and RVEF measured in 3D-ECHO and CMR with a documented tendency towards minimal (although statistically significant) underestimation of 3D-ECHO results [23, 24]. Most of those studies concerned adults [25-29]. The literature comparing results of 3DECHO and CMR in children is much scarcer both in number and resources [27]. It usually addresses specific populations of patients: with congenital or acquired heart disease [30], after heart defect operations (prominently tetralogy of Fallot [31]) or with left ventricular cardiomyopathies [32]. No paper so far has offered data on the comparison between 3D-ECHO and CMR for ventricular function assessment in children with arrhythmia.

Whether impaired LVEF and RVEF are the source or the result of severe arrhythmia, and if ventricular function indeed deteriorates due to arrhythmic disorders, only further studies, especially within the pediatric population diagnosed with arrhythmia in morphologically healthy hearts, will show.

For this reason, systematic, regular, and accurate assessment of ventricular function in children with arrhythmia, even (or maybe especially) idiopathic, is crucial. 3D-ECHO appears to be the 
perfect tool for this purpose and a valuable alternative to CMR in this population [26-28, 33-36].

It is suggested herein, that after initial CMR children with arrhythmias and left ventricular cardiomyopathies can be monitored with 3D-ECHO. CMR can be repeated only in cases of deterioration of ventricular function observed in 3D-ECHO.

\section{Limitations of the study}

In the present study, attention concentrated on assessing the rank of agreement between 3D-ECHO and CMR results in a pediatric population, focusing prominently on arrhythmic patients in whom imaging was both necessary and technically difficult. Because the population analyzed was small, the problem calls for further study, especially in children with arrhythmia. Comparisons to a control group of healthy children would provide statistical clarity on the subject of potential ventricular dysfunction in patients with arrhythmia preliminarily classified as idiopathic, but it is difficult to gather a group of healthy children in whom CMR was used. One should also keep in mind that children with idiopathic arrhythmia might show a tendency towards deterioration of ventricular function in adulthood; therefore, follow-up assessment can be fruitful.

\section{Conclusions}

Three-dimensional echocardiography assessment of both LVEF and RVEF in children shows a high significant correlation and agreement with CMR. 3D-ECHO could be a valuable tool in the follow-up of children with arrhythmic disorders requiring regular assessment of ventricular function.

\section{Conflict of interest: None declared}

\section{References}

1. Helbing WA, Ouhlous M. Cardiac magnetic resonance imaging in children. Pediatr Radiol. 2015; 45(1): 20-26, doi: 10.1007/s00247014-3175-x, indexed in Pubmed: 25552387.

2. Barczuk-Falęcka M, Małek ŁA, Roik D, et al. Right ventricular end-systolic area as a simple first-line marker predicting right ventricular enlargement and decreased systolic function in children referred for cardiac magnetic resonance imaging. Clin Radiol. 2018; 73(6): 592.e9-592.e14, doi: 10.1016/j.crad.2018.01.020, indexed in Pubmed: 29519499.

3. Bland JM, Altman DG. Applying the right statistics: analyses of measurement studies. Ultrasound Obstet Gynecol. 2003; 22(1): 85-93, doi: 10.1002/uog.122, indexed in Pubmed: 12858311.

4. Ratcliffe M, Starr N. Patient management exchange: Cardiac arrhythmias in children. J Pediatric Health Care. 2000; 14(3): 0127-0129, doi: 10.1067/mph.2000.106362.
5. Kim SS, Ko SM, Song MG, et al. Assessment of global function of left ventricle with dual-source CT in patients with severe arrhythmia: a comparison with the use of two-dimensional transthoracic echocardiography. Int J Cardiovasc Imaging. 2010; 26(Suppl 2): 213-221, doi: 10.1007/s10554-010-9692-2, indexed in Pubmed: 20798989.

6. Do VB, Tsai WC, Lin YJ, et al. The different substrate characteristics of arrhythmogenic triggers in idiopathic right ventricular outflow tract tachycardia and arrhythmogenic right ventricular dysplasia: new insight from noncontact mapping. PLoS One. 2015; 10(10): e0140167, doi: 10.1371/journal.pone.0140167, indexed in Pubmed: 26488594.

7. Hennig A, Salel M, Sacher F, et al. High-resolution three-dimensional late gadolinium-enhanced cardiac magnetic resonance imaging to identify the underlying substrate of ventricular arrhythmia. Europace. 2018; 20(FI2): f179-f191, doi: 10.1093/europace/ eux278, indexed in Pubmed: 29069369.

8. Raymond-Paquin A, Nattel S, Wakili R, et al. Mechanisms and clinical significance of arrhythmia-induced cardiomyopathy. Can J Cardiol.2018; 34(11): 1449-1460, doi: 10.1016/j.cjca.2018.07.475, indexed in Pubmed: 30404750.

9. Li KaH, Bazoukis G, Liu T, et al. Arrhythmogenic right ventricular cardiomyopathy/dysplasia (ARVC/D) in clinical practice. J Arrhythm. 2018; 34(1): 11-22, doi: 10.1002/joa3.12021, indexed in Pubmed: 29721109.

10. Chungsomprasong P, Hamilton R, Luining W, et al. Left ventricular function in children and adolescents with arrhythmogenic right ventricular cardiomyopathy. Am J Cardiol. 2017; 119(5): 778-784, doi: 10.1016/j.amjcard.2016.11.020, indexed in Pubmed: 28040191.

11. Steinmetz M, Krause U, Lauerer P, et al. Diagnosing ARVC in pediatric patients applying the revised task force criteria: importance of imaging, 12-lead ECG, and genetics. Pediatr Cardiol. 2018 [Epub ahead of print], doi: 10.1007/s00246-018-1875-y, indexed in Pubmed: 29754204.

12. Mast TP, James CA, Calkins H, et al. Evaluation of structural progression in arrhythmogenic right ventricular dysplasia/cardiomyopathy. JAMA Cardiol. 2017; 2(3): 293-302, doi: 10.1001/ jamacardio.2016.5034, indexed in Pubmed: 28097316.

13. Mast TP, Taha K, Cramer MJ, et al. The prognostic value of right ventricular deformation imaging in early arrhythmogenic right ventricular cardiomyopathy. JACC Cardiovasc Imaging. 2018 [Epub ahead of print], doi: 10.1016/j.jcmg.2018.01.012, indexed in Pubmed: 29550307.

14. Sarvari SI, Haugaa KH, Anfinsen OG, et al. Right ventricular mechanical dispersion is related to malignant arrhythmias: a study of patients with arrhythmogenic right ventricular cardiomyopathy and subclinical right ventricular dysfunction. Eur Heart J. 2011; 32(9): 1089-1096, doi: 10.1093/eurheartj/ehr069, indexed in Pubmed: 21406439.

15. Pietrzak R, Werner B. Postsystolic shortening is associated with altered right ventricular function in children after tetralogy of Fallot surgical repair. PLoS One. 2017; 12(1): e0169178, doi: 10.1371/journal.pone.0169178, indexed in Pubmed: 28046050.

16. Lipczyńska M, Szymański P, Kumor M, et al. Global longitudinal strain may identify preserved systolic function of the systemic right ventricle. Can J Cardiol. 2015; 31(6): 760-766, doi: 10.1016/j.cjca.2015.02.028, indexed in Pubmed: 25935885.

17. Sano H, Tanaka H, Motoji $\mathrm{Y}$, et al. Right ventricular function and right-heart echocardiographic response to therapy predict longterm outcome in patients with pulmonary hypertension. Can J Cardiol. 2015; 31(4): 529-536, doi: 10.1016/j.cjca.2015.01.027, indexed in Pubmed: 25840102.

18. Rudski L, Lai W, Afilalo J, et al. Guidelines for the Echocardiographic Assessment of the Right Heart in Adults: A Report from 
the American Society of Echocardiography. J Am Soc Echocardiogr. 2010; 23(7): 685-713, doi: 10.1016/j.echo.2010.05.010.

19. Lang RM, Badano LP, Mor-Avi V, et al. Recommendations for cardiac chamber quantification by echocardiography in adults: an update from the American Society of Echocardiography and the European Association of Cardiovascular Imaging. J Am Soc Echocardiogr. 2015; 28(1): 1-39.e14, doi: 10.1016/j.echo.2014.10.003, indexed in Pubmed: 25559473.

20. Velasco O, Beckett MQ, James AW, et al. Real-Time three-dimensional echocardiography: characterization of cardiac anatomy and function-current clinical applications and literature review update. Biores Open Access. 2017; 6(1): 15-18, doi: 10.1089/ biores.2016.0033, indexed in Pubmed: 28303211.

21. Badano LP, Boccalini F, Muraru D, et al. Current clinical applications of transthoracic three-dimensional echocardiography. J Cardiovasc Ultrasound. 2012; 20(1): 1-22, doi: 10.4250/ jcu.2012.20.1.1, indexed in Pubmed: 22509433.

22. Surkova E, Muraru D, Aruta P, et al. Current clinical applications of three-dimensional echocardiography: when the technique makes the difference. Curr Cardiol Rep. 2016; 18(11): 109, doi: 10.1007/s11886-016-0787-9, indexed in Pubmed: 27628295.

23. Shimada YJ, Shiota T. A meta-analysis and investigation for the source of bias of left ventricular volumes and function by three-dimensional echocardiography in comparison with magnetic resonance imaging. Am J Cardiol. 2011; 107(1): 126-138, doi: 10.1016/j.amjcard.2010.08.058, indexed in Pubmed: 21146700.

24. Shimada YJ, Shiota M, Siegel RJ, et al. Accuracy of right ventricular volumes and function determined by three-dimensional echocardiography in comparison with magnetic resonance imaging: a meta-analysis study. J Am Soc Echocardiogr. 2010; 23(9): 943-953, doi: 10.1016/j.echo.2010.06.029, indexed in Pubmed: 20797527.

25. Hoffmann R, Barletta G, von Bardeleben S, et al. Analysis of left ventricular volumes and function: a multicenter comparison of cardiac magnetic resonance imaging, cine ventriculography, and unenhanced and contrast-enhanced two-dimensional and three-dimensional echocardiography. J Am Soc Echocardiogr. 2014; 27(3): 292-301, doi: 10.1016/j.echo.2013.12.005, indexed in Pubmed: 24440110.

26. Hamilton-Craig CR, Stedman K, Maxwell R, et al. Accuracy of quantitative echocardiographic measures of right ventricular function as compared to cardiovascular magnetic resonance. Int J Cardiol Heart Vasc. 2016; 12: 38-44, doi: 10.1016/j.ijcha.2016.05.007, indexed in Pubmed: 28616541.

27. Lu X, Nadvoretskiy V, Bu L, et al. Accuracy and reproducibility of real-time three-dimensional echocardiography for assessment of right ventricular volumes and ejection fraction in children. J Am Soc Echocardiogr. 2008; 21(1): 84-89, doi: 10.1016/j. echo.2007.05.009, indexed in Pubmed: 17628408.

28. Park JB, Lee SP, Lee JH, et al. Quantification of right ventricular volume and function using single-beat three-dimensional echocardiography: a validation study with cardiac magnetic resonance. J Am Soc Echocardiogr. 2016; 29(5): 392-401, doi: 10.1016/j. echo.2016.01.010, indexed in Pubmed: 26969137.

29. Jenkins C, Chan J, Bricknell K, et al. Reproducibility of right ventricular volumes and ejection fraction using real-time threedimensional echocardiography: comparison with cardiac MRI. Chest. 2007; 131(6): 1844-1851, doi: 10.1378/chest.06-2143, indexed in Pubmed: 17400663.

30. Balluz R, Liu L, Zhou X, et al. Real time three-dimensional echocardiography for quantification of ventricular volumes, mass, and function in children with congenital and acquired heart diseases. Echocardiography. 2013; 30(4): 472-482, doi: 10.1111/ echo.12132, indexed in Pubmed: 23551607.

31. D'Anna C, Caputi A, Natali B, et al. Improving the role of echocardiography in studying the right ventricle of repaired tetralogy of Fallot patients: comparison with cardiac magnetic resonance. Int J Cardiovasc Imaging. 2018; 34(3): 399-406, doi: 10.1007/ s10554-017-1249-1, indexed in Pubmed: 28988308.

32. Windram JD, Dragelescu A, Benson L, et al. Myocardial dimensions in children with hypertrophic cardiomyopathy: a comparison between echocardiography and cardiac magnetic resonance imaging. Can J Cardiol. 2016; 32(12): 1507-1512, doi: 10.1016/j. cjca.2016.06.014, indexed in Pubmed: 27789109.

33. Laser KT, Horst JP, Barth P, et al. Knowledge-based reconstruction of right ventricular volumes using real-time three-dimensional echocardiographic as well as cardiac magnetic resonance images: comparison with a cardiac magnetic resonance standard. J Am Soc Echocardiogr. 2014; 27(10): 1087-1097, doi: 10.1016/j. echo.2014.05.008, indexed in Pubmed: 24969839.

34. Knight DS, Grasso AE, Quail MA, et al. Accuracy and reproducibility of right ventricular quantification in patients with pressure and volume overload using single-beat three-dimensional echocardiography. J Am Soc Echocardiogr. 2015; 28(3): 363-374, doi: 10.1016/j.echo.2014.10.012, indexed in Pubmed: 25499839.

35. Nagata Y, Wu VCC, Kado Y, et al. Prognostic value of right ventricular ejection fraction assessed by transthoracic 3D echocardiography. Circ Cardiovasc Imaging. 2017; 10(2), doi: 10.1161/ CIRCIMAGING.116.005384, indexed in Pubmed: 28174197.

36. Kamińska H, Werner B. Three-dimensional echocardiography in the assessment of ventricular function in children: pros, cons, and hopes. Kardiol Pol. 2019; 77(1): 12-17, doi: 10.5603/ KP.a2018.0244, indexed in Pubmed: 30575007. 